\title{
Formación de Profesores de Ciencias: Una Perspectiva basada en la Historia y Filosofía de la Ciencia
}

\author{
Mansoor Niaz*
}

Artículo recibido: 31-05-2011 y aprobado: 15-06-2011

Formation of Science Professors: A History and Philosophy of Science Perspective

Resumen: El objetivo de este artículo es de reseñar algunos de los experimentos más importantes de los últimos cien años, dentro de una perspectiva crítica de la historia y filosofía de la ciencia. El análisis de los experimentos muestra que el desarrollo científico es muy complejo e involucra interpretaciones alternativas de los datos experimentales, lo que inevitablemente produce conflictos y controversias entre los científicos. Muchos de estos conflictos requieren la intervención de la comunidad científica para su resolución y a veces tardan largos periodos de tiempo. Una revisión bibliográfica de la enseñanza de la ciencia muestra que la mayoría de las/los profesoras(es) de ciencia y textos ignoran estos aspectos del desarrollo científico. Se sugiere que la inclusión de una perspectiva basada en la historia y filosofía de la ciencia puede facilitar una mejor comprensión de la ciencia y asimismo motivar a los estudiantes y los profesores de ciencia, para seguir haciendo la ciencia.

Palabras-clave: Profesores de ciencias, Historia y filosofía de la ciencia, Experimentos, Conflictos, Controversias, Presuposiciones, Método científico, Textos
Abstract: The objective of this article is to review some of the most important experiments of the last one hundred years within a critical perspective of history and philosophy of science. An analysis of the experiments reveals that scientific development is very complex and involves alternative interpretations of experimental data that inevitably leads to conflicts and controversies among scientists. Many of these conflicts require the intervention of the scientific community for their resolution and at times last for long periods of time. A review of the literature in science education reveals that a majority of science teachers and textbooks ignore these aspects of scientific development. It is suggested that the inclusion of a perspective based on history and philosophy of science can facilitate a better comprehension of science and at the same time motivate students and teachers of science to keep doing science.

Key words: Professors of science, History and philosophy of science, Experiments, Conflicts, Controversies, Presuppositions, Scientific method, Textbooks.

Grupo de Epistemología de la Ciencia. Departamento de Química, Universidad de Oriente 


\section{Introducción}

Muchos de los docentes, textos y planes curriculares creen que para la formación de los profesores de ciencias lo más importante es la realización de los experimentos. Esta conceptualización lleva a los estudiantes a memorizar los detalles experimentales, y luego la elaboración de las teorías científicas como una consecuencia lógica del proceso científico, quiere decir:

experimentos $\rightarrow$ leyes $\rightarrow$ teorías (para algunos docentes las teorías vienen antes de las leyes). Esta conceptualización carece de una comprensión epistemológica de la naturaleza de la ciencia ( $\mathrm{NdC}$ ), que abarca entre otros los siguientes aspectos (Niaz y Maza, 2011): 1) Por qué un científico hace un experimento; 2) Los mismos datos experimentales pueden ser interpretado de maneras distintas por diferentes científicos; 3) Antes de realizar un experimento los científicos elaboran su marco teórico (presuposiciones) y tiene una idea previa de lo que serán los resultados; 4) Toda observación está impregnada de una teoría; 5) $\mathrm{Al}$ obtener resultados no esperados (anómalos) los científicos no abandonan su marco teórico de inmediato; 6) El progreso científico está caracterizado por conflictos, competencias, inconsistencias, y controversias entre teorías rivales; 7) Las teorías científicas son tentativas; 8) Las teorías y leyes juegan papeles diferentes y al tener evidencia adicional una teoría no se convierte en una ley (o viceversa); 9) El método científico no juega un papel importante en la investigación científica; 10) Los experimentos son importantes pero sus interpretaciones lo son aún más.

A continuación se presentan varios experimentos importantes en la his- toria de la ciencia, aproximadamente últimos cien años, para demostrar que el desarrollo científico es muy diferente de lo que se presenta en los textos y programas de ciencias, los cuales a veces son seguido a pie de la letra por los profesores de ciencia. A pesar ciertas discrepancias hay suficiente consenso alrededor de estos aspectos del NdC, dentro de la comunidad de la enseñanza de la ciencia.

\section{Experimento de Michelson-Morley (1887) para determinar la velocidad de la Tierra con respecto al éter}

Este experimento es uno de los más difíciles de entender y controversial en la historia de la ciencia, y reportó un resultado nulo, quiere decir no hay una velocidad de la Tierra con respecto el éter (Holton, 1969a; 1969b). Este resultado no fue lo que esperaban muchos de los científicos creyentes en la teoría del éter y siguieron repitiendo el experimento hasta 1927 con la esperanza de obtener resultados positivos (Lakatos, 1970). Por otro lado, muchos científicos reconocidos (incluyendo Robert Millikan y por supuesto el mismo Michelson) establecieron una relación genética entre el experimento y la teoría de la relatividad especial (TRE) de Einstein. En otras palabras de acuerdo con estos científicos Einstein presentó su TRE en 1905, basándose en los resultados del experimento de Michelson-Morley. Algunos de los textos de física general todavía sugieren que este experimento ayudó a Einstein a formular la teoría de la relatividad en 1905 (Brush, 2000). No obstante, la paradoja es que: Einstein aclaró que él no había oído del experimento antes de formular su teoría. 


\section{Experimento de Thomson de Rayos Catódicos (1897)}

Cuando J.J. Thomson comenzó sus experimentos el estaba consciente de la controversia con respecto a la naturaleza de los rayos catódicos, la cual giraba en torno a la pregunta: ¿Eran partículas u ondas en el éter? (Falconer, 1987). Para resolver este problema Thomson determinó la relación carga/masa (e/m) utilizando diferentes gases y electrodos en el tubo de rayos catódicos. Los resultados indicaron que la relación $(\mathrm{e} / \mathrm{m})$ era constante para los diferentes gases. Basándose en estos resultados, muchos de los textos de química y física dicen que Thomson descubrió el electrón en 1897 (Niaz, 1998). Es interesante notar que G. FitzGerald (1897), un colega de Thomson, aceptó la existencia de electrones pero a la vez cuestionó si los electrones eran constituyentes de todos los átomos. El trabajo de FitzGerald se publicó en el mismo número (Mayo 21, 1897) de la revista The Electrician, en el cual Thomson (1897) publicó la versión preliminar de su artículo. En una reconstrucción histórica reciente Arabatzis (2006) ha demostrado que el electrón no fue descubierto por Thomson en $1897 \mathrm{y}$ ni posteriormente, sino que fue incorporado gradualmente en la ontología de la química y física.

\section{Experimento de dispersión de partículas alfa (1911)}

De acuerdo con los experimentos de Rutherford (1911), solo un número muy pequeño de partículas alfa se desvían en ángulos grandes. Los experimentos de Rutherford lo llevaron a proponer el modelo 'nuclear' del átomo (basándose en la hipótesis de dispersión simple que requiere la concentración de la carga positiva en una parte diminutiva del átomo). Thomson estaba al tanto de los experimentos de Rutherford aun antes de su publicación, y esto produjo una rivalidad y por ende un conflicto con el modelo atómico de Thomson. Estos conflictos fueron discutidos tanto en la literatura especializada como en congresos científicos por Thomson y Rutherford (Wilson, 1983). Es importante notar que Thomson también hizo experimentos con la desviación de las partículas alfa y llegó a rechazar el modelo 'nuclear' de Rutherford. Reinterpretando los datos basándose en su modelo de 'budín con pasas' (así descrito en los textos, aunque el modelo era mucho más complejo), Thomson explicó que las partículas alfa al entrar al átomo chocaban con varias cargas positivas sucesivamente y al final se desviaban en un ángulo grande (hipótesis de dispersión múltiple). La comunidad científica tuvo que enfrentar una dura prueba, en vista de que dos científicos reconocidos explicaban los mismos datos experimentales de dos maneras diferentes: a) Hipótesis de dispersión simple (Rutherford) y b) Hipótesis de dispersión múltiple (Thomson). Es interesante notar que hoy nuestros textos, al describir el experimento de desviación de partículas alfa de Rutherford concluyen que basándose en los datos experimentales, el modelo 'nuclear' era una "conclusión lógica” (Niaz, 1998; 2009). No obstante, Thomson considerado como un 'maestro en la construcción de los modelos atómicos' no vio una relación 'lógica'. La rivalidad entre Thomson y Rutherford llegó a un nivel tal que Rutherford acusó a uno de los colaboradores de Thomson de falsear los datos experimentales para continuar apoyando el modelo atómico de Thom- 
son (Wilson, 1983). ¿Cómo explica Ud., estimado profesor, que dos científicos célebres, con los mismos resultados experimentales propusieron dos modelos (teorías) diferentes. Esto demuestra que ante una nueva evidencia empírica, los científicos no cambian su teoría inicial fácilmente (Niaz, 2009).

\section{Experimentos relacionados con los espectros de los elementos}

N. Bohr arribó a Manchester en marzo de 1912 y luego de realizar algunos experimentos sobre radioactividad, comenzó a trabajar en un proyecto para cuantizar el modelo atómico de E. Rutherford. En julio de 1912 presentó a la consideración de Rutherford un primer proyecto sobre la constitución de átomos y moléculas. Para marzo de 1913 Bohr lo sometió nuevamente a la consideración de Rutherford, después de lo cual fue publicado en la revista Philosophical Magazine. El aspecto más importante de este artículo (Bohr, 1913) fue una plausible explicación de la estabilidad paradójica del modelo atómico de Rutherford. No obstante, la mayoría de los textos de química y física consideran que la principal contribución de Bohr es la explicación de las series de Balmer y Paschen de las líneas espectrales de hidrógeno. La paradoja es: Bohr reconoció que antes de escribir la primera versión de su artículo en 1912, él no sabía del trabajo de Balmer (Niaz, 1998). Este episodio es importante por el hecho de que muchos de los filósofos positivistas argumentaban que los datos experimentales sobre los espectros fueron primero comprendido a través de la fórmula de Balmer y luego explicado por la teoría de Bohr. Los filósofos positivistas en general creen que el desarrollo científico sigue la secuencia: datos experimentales (espectros de los elementos) $\rightarrow$ Ley de Balmer $\rightarrow$ Teoría de Bohr.

\section{Experimento de la gota de aceite de Millikan}

La determinación de la carga eléctrica fundamental (electrón) por parte de R.A. Millikan (experimento de la gota de aceite) es otro ejemplo que demuestra la relación compleja que existe entre los experimentos y el marco teórico previo del científico (presuposiciones). La reconstrucción histórica (Holton, 1978) muestra que hubo una controversia entre Millikan (Universidad de Chicago) y otro científico (F. Ehrenhaft, Universidad de Viena) que duró casi veinte años (1909-1929). Los datos experimentales de Millikan y Ehrenhaft eran muy parecidos. No obstante, sus marcos teóricos e interpretaciones eran totalmente diferentes. Basándose en su marco teórico previo (antes de realizar los experimentos), Millikan formuló la existencia de la carga eléctrica fundamental (electrón). Por el otro lado, Ehrenhaft formuló la existencia de sub-electrones. Es importante notar que Ehrenhaft pertenecía a la escuela de anti-atomistas célebres, tales como E. Mach y W. Ostwald, y eso explica en parte su negativa a aceptar la naturaleza de una partícula universal con carga (quiere decir el electrón). Esto plantea un interrogante, si los datos experimentales de los dos científicos eran muy parecidos, ¿cómo es posible que sus interpretaciones hayan sido tan diferentes? La respuesta es simple, aunque difícil de creer. Millikan para sostener su marco teórico (la existencia del electrón) descartó, un poco arbitrariamente, varias medidas experimentales (en algunos experimentos, casi el 59\% de 
las gotas estudiadas). Ehrenhaft, siendo un experimentalista riguroso dejó que sus datos "le dictaran" la teoría. Es interesante notar que cuando Millikan (siendo 10 años mayor) comenzó su carrera, Ehrenhaft ya formaba parte de la elite científica europea (Universidad de Viena). Hoy, después de casi noventa años, la comunidad científica y los textos de física y química no solamente ignoran a Ehrenhaft (Niaz, 2000), sino que no reconocen lo difícil que fue la interpretación de los experimentos que condujeron a Millikan hacia la determinación de la carga eléctrica fundamental (para más detalles ver Niaz, 2005; 2009).

\section{Experimentos relacionados con el efecto fotoeléctrico (1916)}

Robert Millikan fue responsable de innovar y determinar la constante de Planck (h) basándose en la ecuación de efecto fotoeléctrico de Einstein. A pesar de este éxito, Millikan aceptaba la teoría clásica de radiación (éter) y rechazaba la teoría cuántica de radiación de Einstein. Es interesante notar que Millikan no solamente dudaba de las ideas de Einstein, sino que consideró que estas ideas eran insostenible y la teoría misma temeraria. Holton (1999) ha descrito el dilema de Millikan en términos de su presuposición: "A lo que hoy día referimos como el fotón, era en la opinión de Millikan, 'atrevido, por no decir una hipótesis imprudente' --- era imprudente porque contradecía los conceptos clásicos, tales como el fenómeno de propagación de la luz y los hechos de interferencia" (p. 232). La hipótesis de Einstein no fue aceptada por la comunidad científica (incluyendo Planck) por muchos años. Millikan presentó evidencia experimental para comprobar la ecuación de Einstein, y el mismo tiempo rechazaba la teoría de cuanto de Einstein. Esto demuestra que las ideas noveles no son fáciles de aceptar, debido a la presuposiciones que tienen los científicos y por ende también los estudiantes. Es interesante notar que muchos de los textos de física general dicen que Millikan comprobó la teoría cuántica de Einstein (Niaz, Klassen, McMillan, y Metz, 2010). Se puede concluir que ninguna evidencia experimental puede proporcionar la prueba concluyente para una teoría, de allí que aun cuando Millikan presentó una demostración experimental, esto no fue suficiente para que la teoría de Einstein fuera aceptada dentro de la comunidad científica.

\section{Experimentos de eclipse de sol en 1919 como evidencia para la teoría de relativi- dad general de Einstein}

La Sociedad Astronómica Real de Inglaterra organizó dos expediciones para observar el eclipse de sol en 1919. El objetivo de la expedición era obtener evidencia experimental para la teoría general de la relatividad de Einstein. Una de las expediciones se trasladó a la isla de Principe (costa oeste de África) y la otra a Sobral (noroeste de Brasil). El grupo de Principe fue dirigido por A.S. Eddington, el Astrónomo Real de Inglaterra, y una de las pocas personas que se especializaba en la teoría de la relatividad de Einstein, en 1919. Una revisión bibliográfica muestra que los experimentos eran difíciles de realizar y la interpretación de los datos experimentales extremadamente difícil. La controversia con respecto a la interpretación de los datos experimentales continuó por varias décadas (p.e., Brush, 1999; Collins \& Pinch, 1998). Antes de realizar los ex- 
perimentos en 1919 Eddington esperaba tres posibles resultados:

a) La luz atraviesa el campo de gravitación del sol sin ninguna desviación

b) Desviación de 0,87" (concuerda con la teoría de Newton)

c) Desviación de 1,75" (concuerda con la teoría de Einstein)

Resultados experimentales obtenidos fueron los siguientes:

1) Telescopio astrográfico Sobral $=0,87^{\prime \prime}$

2) Telescopio de 4 pulgadas Sobral = $1,98 "$

3) Telescopio astrográfico Principe = $1.61^{\prime \prime}$

En vista de que ninguno de los tres telescopios facilitó un valor de la desviación de la luz cercano a la predicción de Einstein (quiere decir 1.75"), no era fácil llegar a una conclusión definitiva. No obstante, la paradoja es que: Eddington concluyó que los experimentos suministraron evidencia empírica para apoyar la teoría de Einstein, por el hecho de que la desviación de 1,98" estaba muy cerca de la predicción de 1,75" hecha por Einstein (Dyson, Eddington y Davidson, 1919). Un lector curioso puede preguntar: Qué pasó con los datos del telescopio astrográfico Sobral (0.87", coincidente con la teoría de Newton)? La respuesta de Eddington y su grupo fue la siguiente: Este día en Sobral estaba muy nublado y las fotografías salieron muy borrosas (para detalles ver Niaz, 2009, capítulo 9). Esto demuestra claramente que las presuposiciones sobre la teoría de relatividad de Eddington, inclinaron la balanza a favor de Einstein. Un grupo de filósofos ha resumido el dilema de comprender el desarrollo científico en los siguientes términos:

Lo que la ciencia dice con respecto a lo que es el mundo en un momento dado, es afectado por las ideas humanas, opciones, expectativas, prejuicios, creencias y presuposiciones vigente en este momento (Machamer, Pera y Baltas, 2000, p. 6).

\section{Experimentos para aislar los quarks}

Muchos de los cursos de ciencias y textos enfatizan que la partícula fundamental con carga es el electrón. No obstante, por lo menos desde la década de los años sesenta los físicos han estado buscando las cargas fraccionales (quarks). Hoy el aislamiento de los quarks constituye un problema a resolver en la frontera de la ciencia moderna. Entre muchos otros investigadores, Martin Perl (Premio Nobel de Física, 1995) ha estado activo, desde hace muchos años en diseñar experimentos complejos para el aislamiento de los quarks y la determinación de su carga (se supone que sea $1 / 3,1 / 4$ o hasta $1 / 10$ de la carga de un electrón). Dadas las dificultades de diseñar experimentos y a la vez su interpretación, Perl ha elaborado una filosofía de experimentos especulativos para aislar los quarks:

"Selección del diseño de los experimentos especulativos, muchas veces no se puede realizar simplemente basándose en puro razonamiento. El experimentador muchas veces tiene que basar su decisión parcialmente en lo que se siente que es correcto, parcialmente en la tecnología que le gusta y en algunos aspectos de las especulaciones" (Perl y Lee, 1997, p. 699). 
Esto demuestra claramente la importancia de los experimentos especulativos, sobre todo, cuando el científico esta abrumado con dificultades, el futuro de la investigación no se puede predecir y la posibilidad de lograr el éxito está en juego por la competencia de los pares. Es importante señalar que la competencia entre diferentes grupos de científicos es una característica muy importante del desarrollo científico, en vista de que facilita la convalidación de los resultados por medios diferentes.

\section{Conclusión}

En este artículo he reseñado algunos de los experimentos más importantes de los últimos cien años, para demostrar como los científicos hacen ciencia. Asimismo, he tratado de interpretar los experimentos dentro de una perspectiva basada en la historia y filosofía de la ciencia. Sería oportuno preguntar si nuestros profesores de ciencias y los textos presentan la ciencia tal como lo practican los científicos (Niaz, 2010).

\section{Bibliografía}

Arabatzis, T. (2006). Representing electrons: A biographical approach to theoretical entities. Chicago: University of Chicago Press.

Bohr, N. (1913). On the constitution of atoms and molecules, Part I. Philosophical Magazine, 26 (Series 6), 1-25.

Brush, S.G. (1999). Why was relativity accepted? Physics in Perspective, 1(2), 184-214.

Brush, S.G. (2000). Thomas Kuhn as a historian of science. Science \& Education, 9 (1-2), 39-58.

Collins, H., \& Pinch, T. (1998). The Golem: what you should know about science
Una revisión bibliográfica de la enseñanza de la ciencia en diferentes partes del mundo muestra que la mayoría de los docentes y textos ignoran estos aspectos del desarrollo científico (Galagovsky, 2011; Niaz, 2011). Es importante que nuestros estudiantes y profesores de ciencia estén conscientes de las dificultades involucradas en el desarrollo de la ciencia y que éste requiere no un recetario (método científico) para realizar los experimentos sino que mucha creatividad, imaginación, perseverancia, y la habilidad para 'especular' y soñar. Se sugiere que la inclusión de los detalles de cómo los científicos practican la ciencia puede motivar a nuestros estudiantes a seguir la carrera científica y así pensar que ellos también podrían ser los protagonistas del desarrollo científico en el futuro.

Para finalizar, es interesante notar que Einstein en una conversación con Heisenberg en Abril de 1926 afirmó: "Solo la teoría decide lo que uno puede observar".

(2 ed). Cambridge: Cambridge University Press.

Dyson, F.W., Eddington, A.S., \& Davidson, C. (1920). A determination of the deflection of light by the sun's gravitational field, from observations made at the total eclipse of May 29, 1919. Royal Society Philosophical Transactions, 220, 291-333.

Falconer, I. (1987). Corpuscles, electrons, and cathode rays: J. J. Thomson and the 'discovery of the electron.' British Journal for the History of Science, 20, 241-276.

FitzGerald, G. (1897). Dissociation of atoms. The Electrician, 39, 103. 
Galagovsky, L. (2011) Coordinadora. Didáctica de las ciencias naturales: El caso de los modelos científicos. Buenos Aires: Lugar Editorial (Colección Nuevos Paradigmas).

Holton, G. (1969a). Einstein and 'crucial' experiments. American Journal of Physics, 37(10), 968-982.

Holton, G. (1969b). Einstein, Michelson, and the 'crucial' experiment. Isis, 60, 133-197.

Holton, G. (1978). Subelectrons, presuppositions, and the Millikan-Ehrenhaft dispute. Historical Studies in the Physical Sciences, 9, 161-224.

Holton, G. (1999). R.A. Millikan's Struggle with the Meaning of Planck's Constant. Physics in Perspective, 1, 231-237.

Lakatos, I. (1970). Falsification and the methodology of scientific research programmes. In I. Lakatos \& A. Musgrave (Eds.), Criticism and the growth of knowledge (pp. 91-195). Cambridge: Cambridge University Press.

Machamer, P., Pera, M., \& Baltas, A. (2000). Scientific controversies: An introduction. In P. Machamer, M. Pera \& A. Baltas (Eds.), Scientific controversies: Philosophical and historical perspectives (pp. 3-17). New York: Oxford University Press.

Niaz, M. (1998). From cathode rays to alpha particles to quantum of action: a rational reconstruction of structure of the atom and its implications for chemistry textbooks. Science Education, 82, 527-552.

Niaz, M. (2000). The oil drop experiment: A rational reconstruction of the Millikan-Ehrenhaft controversy and its implications for chemistry textbooks. Journal of Research in Science Teaching, 37, 480-508.
Niaz, M. (2005). An appraisal of the controversial nature of the oil drop experiment: Is closure possible? British Journal for the Philosophy of Science, 56(4), 681-702.

Niaz, M. (2009). Critical appraisal of physical science as a human enterprise: Dynamics of scientific progress. Dordrecht, The Netherlands: Springer.

Niaz, M. (2010). Are we teaching science as practiced by scientists? American Journal of Physics, 78(1), 5-6.

Niaz, M. (2011). Innovating science teacher education: A history and philosophy of science perspective. New York: Routledge.

Niaz, M., Klassen, S., McMillan, B., y Metz, D. (2010). Reconstruction of the history of the photoelectric effect and its implications for general physics textbooks. Science Education, 94, 903-931.

Niaz, M., y Maza, A. (2011). Nature of science in general chemistry textbooks. Dordrecht, The Netherlands: SpringerBriefs in Education.

Perl, M.L., y Lee, E.R. (1997). The search for elementary particles with fractional electric charge and the philosophy of speculative experiments. American Journal of Physics, 65, 698-706.

Rutherford, E. (1911). The scattering of alpha and beta particles by matter and the structure of the atom. Philosophical Magazine, 21, 669-688.

Thomson, J.J. (1897). Cathode rays. Philosophical Magazine, 44, 293-316.

Wilson, D. (1983). Rutherford: Simple genius. Cambridge, MA: MIT Press. 